\title{
The Developmental Experiences of Expert Athletes in Sports with Different Levels of Risk
}

\author{
${ }^{1}$ Negin Atefineya, ${ }^{1}$ Rokhsareh Badami* ${ }^{2}$ Jared M. Porter \\ ${ }^{1}$ Department of Physical Education and Sport Science, Isfahan (Khorasgan) Branch, Islamic Azad \\ University, Isfahan, Iran. ${ }^{2}$ Department of Kinesiology, Recreation, and Sport Studies, The University of \\ Tennessee, Knoxville, State of Tennessee, USA.
}

Submitted 24 March 2020; Accepted in final form 06 July 2020.

\begin{abstract}
Background. Recent research has proposed that environmental factors affect the development of expertise. Objectives. The present study aimed to compare the history of training activities amongst expert athletes of different sport disciplines regarding the level of risk. Methods. Participants included 187 athletes from six sports disciplines, which were allocated to "low-risk," "medium risk," or "high-risk" groups based on the level of physical risk placed on the participating athletes. Participants completed a developmental history of athletes' questionnaire. Results. Findings revealed a significant difference amongst groups in terms of the mean age of beginning the sport $(\mathrm{p}<0.001)$. Additionally, there was no significant difference between the total hours of deliberate practice and physical preparation amongst the three groups $(\mathrm{p}>0.05)$. However, the low-risk group had an enormous amount of deliberate practice $(\mathrm{p}<$ $0.001)$ and physical preparation activities $(\mathrm{p}=0.002)$ in the 6 to 12-year age band, and the medium-risk and high-risk sports groups had an enormous amount of deliberate practice $(\mathrm{p}=0.01)$ and physical preparation activities $(\mathrm{p}=0.003)$ in the age band of above 16 years. Conclusion. The present study provides useful information on the expertise acquisition process in different sport disciplines regarding the amount of practice relative to risk level.
\end{abstract}

\section{KEYWORDS: Deliberate Practice, Elite, Exercise, Development, Training.}

\section{INTRODUCTION}

Genetic and environmental factors affect sports performance (1). Accordingly, studies conducted on expertise in sport have been divided into four developmental areas, including 1) biological (genetic) description of expertise development in sport $(2,3), 2)$ training as the primary contributor to expertise development in sport $(4,5), 3)$ the environmental factors influencing expertise development $(6,7)$, and 4) the multifactorial approach which encompasses the previous three factors $(8,9)$. One of the models having been taken into consideration in the area of the amount of practice is Côté's developmental model of sport participation
(DMSP) (9, 10). The DMSP deals with the importance of training patterns in the expertise acquisition process. The DMSP consists of three distinct stages, including sampling years (childhood, 6 to 12 years), specializing years (early adolescence, 13 to 15 years), and investing years (late adolescence, older than 16 years) (11). Based on this model, it is recommended that children in the sampling stage participate in different sport disciplines in the form of deliberate play (12). Deliberate play refers to playing games such as soccer in the backyard or basketball in the street with age-based modified rules. These forms of play are regulated and

*. Corresponding Author:

Rokhsareh Badami, Associate Professor

E-mail: rokhsareh.badami@khuisf.ac.ir 
controlled by children or adults involved in the activity. These activities are inherently motivating and are designed to enhance enjoyment (12). Côté and Hay acknowledged that, in the specializing stage, it is better to limit children's participation in different sport activities, and the activities at this stage should be primarily in the form of deliberate play and deliberate practice (12). Deliberate practice is a kind of training done with the primary aim of improving performance. Coaches control this kind of practice, and the coaches' feedback and interventions are conducted to correct errors and improve motor performance (13). Criteria for deliberate practice are 1) Individualized design of effective practice, 2) Active responses to a task with an explicit goal, immediate feedback, and repetition, and 3) Individualized assessment of skill and design of future practice (4).

In the final stage (i.e., during the investment years), young athletes are usually only committed to one sport and are mainly engaged in deliberate practice. Accordingly, three testable principles have been proposed based on the developmental model of sport participation, including 1) as children, expert athletes participated in various sports rather than specializing in one sport. 2) expert athletes participated in deliberate play during childhood, rather than deliberate practice. 3) and lastly, expert athletes narrowed their participation in one or two sport disciplines during adolescence and adulthood, and mainly participated in deliberate practice, rather than deliberate play in adolescence and adulthood (9).

The philosophy of promoting widespread participation in sport and physical activity during the sampling stage is to create motivation for continued participation in sport-related activities. Kirk (14) states that different learning experiences during childhood not only develop physical capabilities but also increase a sense of competency in sport. During the specializing years, deliberate play allows children to experience different sports in different contexts and further grow sport-related excitement. Deliberate play makes it possible for children to be creative in performing their activities, without being worried about adults (i.e., their coaches and parents) intervening to show them the correct way to perform a skill (11). This is an implicit method for skill development, and these children are less likely to forget what they have learned (11). Finally, during the investing years, young athletes increase the amount of time allocated to deliberate practice and reduce the amount of time allocated to deliberate play as they transition from adolescence into adulthood (15).

Conducting a research study on professional ice hockey players, Soberlak and Côté (16) confirmed these three principles. The findings of their study showed that professional ice hockey players spent more than 10000 hours between the ages of $6-20$ years participating in sport-related activities, out of which they spent about 350 hours engaged in game-like activities in other sports. Moreover, 2200 hours were dedicated to the deliberate practice of sports other than hockey, 3000 hours were invested in organized hockey activities (i.e., deliberate practice), and more than 2400 hours were allocated to playing organized hockey games (17). Another study reported that about $50 \%$ of the total training time was spent participating in deliberate practice in one particular sport. In comparison, the same athletes spent $50 \%$ of their time participating in deliberate practice and deliberate play in other sports.

Nonetheless, it seems that the amount of hours spent on participation in deliberate practice depends on the type of sport (18). For example, triathletes usually reach their peak performance at an older age compared to ice hockey players. Thus they spent more time in their childhood participating in sports other than triathlons (11). Accordingly, it seems that it is difficult to declare the average hours of deliberate practice, deliberate play, and training in other sports each week during the period of sport participation, and it cannot be judged as a general rule. It may depend on the type of sport (9).

One of the systems used to classify sport is based on the degree of risk associated with participation in that particular sport. According to Zuckerman's definition (19) sports disciplines are classified into three categories: low-risk, medium-risk, and high-risk. Low-risk sports are associated with a lower chance of becoming injured. Sports such as jogging, bowling, golf, swimming, and aerobics fall into the low-risk category. Medium-risk sports are sports with the highest chance of becoming injured, but they are not generally associated with the risk of death. Sports such as boxing, karate, and American football are considered as examples of this category of sports. In contrast, high-risk sports are sports with the chance of severe injury or even death. High-risk sports include mountaineering, 
rock climbing, high-altitude parachute jumping, free fall, and in general, situations where individuals have to struggle with the problematic elements of nature.

Considering that the expertise acquisition process probably differs depending on the type of sport, the current study was conducted to compare the history of training activities among expert athletes of the low, medium, and high-risk sport disciplines. The pursuit of this aim is essential to obtaining information on the expertise acquisition process to help in the facilitation of officials in educational planning. It can help parents put their children in a better athletic development path.

\section{MATERIALS AND METHODS}

The experimental protocol was reviewed and approved by an Ethical Participant advising committee. We presented information about the study, purposes, procedures, etc., to all participants at the beginning of the study and obtained the informed consent of each participant.

Participants. The participants of this study consisted of 187 athletes competing at national and international levels $(\mathrm{N}=70$ females and $\mathrm{N}=$ 117 males), aged 25 - 35 years, and from six sports disciplines with different degrees of risk (31 athletes in swimming and 28 in gymnastics as low-risk sport disciplines, 25 athletes in karate and 45 in wushu as medium-risk sport disciplines, and ten athletes in paragliding and 48 in rock climbing as high-risk sport disciplines), who were selected using availability and purposive sampling methods.

Apparatus, Task, and Procedure. Data were collected using the Hopwood developmental history of an athlete's questionnaire (DHAQ) (20). Participants' demographic information, the general pattern of their activities from their childhood until their current age, such as the age of beginning their primary sport discipline, the multiplicity of sports they had participated in, the amount of deliberate practice and play in their primary sport discipline, the amount of physical preparation (e.g., strength development, pilates, or any other exercises they had done to enhance the dimensions of their physical preparation), psychological preparation (e.g., relaxation and visualization exercises), and mental preparation (e.g., watching competitions of their primary sport discipline, reading books related to their primary sport, and watching educational videos) and the amount of deliberate play were assessed in this questionnaire. Then the gathered information was summarized and is reported in tables related to the three age bands of 6-12 years, 13-15 years, and 16 and above years.

Developmental History of Athlete's Questionnaire. Practice history (amount of practice and the type of practice activities) were collected using the Hopwood developmental history of an athlete's questionnaire (DHAQ) (20). The first section of the DHAQ is allocated to demographic data such as gender, date of birth, and level of competition.

The next section assessed the athletes' participation in deliberate play. Deliberate play refers to activities that are not very structured and encompass minimal adult supervision. Participation in deliberate play is optional, and the activities are enjoyable and done by inherent motivation and provide immediate satisfaction (12). Therefore, in this section, participants were asked to recall details of their participation in an enjoyable and informal sports game, which was similar to athletic competitions but embraced modified rules and equipment, and was done by minimal adult supervision. Similar to the approach adopted by Baker et al. (17); Berry, Abernethy, \& Côté (21); Côté and Fraser-Thomas (9); participants identified all the activities in which they were involved fitting this explanation along with the age at which they regularly participated in each activity. The number of months per year and the average number of hours per week during which they were involved in that activity were recorded for each year of their participation in an informal and entertaining sports game.

The next section of the DHAQ is assessed engagement in the athletes' primary sport. First, the athletes were asked to provide general information about their participation in their primary sport. For each year of their participation, athletes recalled the total number of months in which they were involved in their primary sport, the average number of hours per week during which they participated in the training, and the average number of training sessions per week.

The next section assessed is engagement in physical preparation (e.g., strength and conditioning, weights, fitness, pilates, yoga, flexibility). The number of months per year and the average number of hours per week during which they were involved in that activity were recorded for each year of their participation.

The final section of the DHAQ assessed engagement in mental preparation (e.g., working 
with a psychologist, video analysis/ review). The number of months per year and the average number of hours per week during which they were involved in that activity were recorded for each year of their participation.

The validity and reliability of this instrument have been assessed using different methods. For instance, the accuracy between data about the age of beginning the sport and years of participation obtained from various sources such as athletes, their parents, and the resume of each athlete has been reported to be $79 \%$ to $100 \%$ (20). For several sports activities, the agreement between the primary and secondary sources of data has also been strong, with the values of agreement ranging between $88 \%$ and $100 \%$ (20). Although the validity and reliability of recalling the amount of time for participation in deliberate play have only been assessed in a small number of studies, it does not seem to be as strong as the validity and reliability of recalling the amount of time for participation in deliberate practice. For instance, Memmert, Baker, and Bertsch (22) obtained a Pearson correlation coefficient of 0.79 for total hours of participation in deliberate practice. However, the Pearson correlation coefficient for total hours of participation in deliberate play was 0.70 in comparison.

Similarly, Soberlak and Côté (16) indicated the correlation between hours of participation in deliberate play reported by athletes and parents with a $30 \%$ difference compared to a $1 \%$ difference for hours of participation in deliberate practice. Despite lower levels of validity and reliability for recalling hours of participation in deliberate play than those in deliberate practice, the reported values still show an acceptable amount of agreement. In other words, it seems that the athlete's participation in sport-related activities is recalled with acceptable validity and reliability (23). In total, the validity and reliability of recalling the hours of participation in training activities have been quite good and have fluctuated with Pearson correlation coefficients ranging from 0.59 to 0.97 (20).

Data Analysis. Data were analyzed using a one-way analysis of variance (ANOVA) and the phi-coefficient analysis tests via the statistical package for the social sciences (SPSS) software version 23 .

\section{RESULTS}

$17.7 \%$ of the study sample was made up of athletes in swimming, $15.4 \%$ of athletes in gymnastics, $9.7 \%$ of athletes in karate, $25.7 \%$ of athletes in wushu, $4 \%$ of athletes in paragliding, and $27.4 \%$ of athletes in rock climbing. Athletes in swimming and gymnastics, karate and wushu, and paragliding and rock climbing were classified into low-risk, medium-risk, and high-risk sport disciplines.

Table 1 compares the age of beginning the sport among low, medium, and high-risk sport disciplines.

Table 1. Estimation of One-Way ANOVA to Compare the Mean age of Beginning and to reach Peak in the Sport

\begin{tabular}{|c|c|c|c|c|c|}
\hline Variable & $\mathbf{N}$ & Mean & Std. Deviation & $\mathbf{F}$ & $\mathbf{p}$ \\
\hline \multicolumn{6}{|l|}{ Age of deliberate beginning } \\
\hline Low-risk & 59 & 6.64 & 2.83 & 79.348 & $<0.001$ \\
\hline Medium-risk & 70 & 11.11 & 3.10 & & \\
\hline High-risk & 58 & 14.09 & 3.70 & & $<0.001$ \\
\hline Age of reaching the peak & & & & 25.464 & \\
\hline Low-risk & 59 & 16.87 & 4.13 & & \\
\hline Medium-risk & 70 & 21.78 & 3.75 & & \\
\hline High-risk & 58 & 22.32 & 4.31 & & \\
\hline
\end{tabular}

Values in Table 1 revealed that the mean age of beginning the sport is lowest in low-risk sport disciplines, and most significant in high-risk sport disciplines. Based on the results of the one-way ANOVA, there was a significant difference between sport disciplines in terms of the mean age of beginning the sport $(\mathrm{p}<0.001)$. Post hoc analyses using the Scheffe post hoc criterion for significance indicate that the mean start age of participants in low-risk sport disciplines was significantly lower than that in both medium-risk and high-risk sport disciplines $(\mathrm{p}<0.001)$. Also, this means it was significantly lower in medium risk sport disciplines in comparison to that of high-risk sport disciplines $(\mathrm{p}<0.001)$.

Table 2 shows the frequency of athletes in low, medium, and high-risk sport disciplines regarding the multiplicity of sports they had participated in.

The values in Table 2 reveal that $61.7 \%$ of the athletes in low-risk sports were single-sport 
athletes, while $37.3 \%$ participated in multiple sports. Also, 52.9\% of the athletes in medium-risk sports reported being single-sport athletes, and $47.1 \%$ were multi-sport athletes. Additionally, $37.9 \%$ of those in high-risk sports were singlesport athletes, whereas $62.1 \%$ were multi-sport athletes. Results of the chi-square test revealed that there was a significant relationship between sport discipline and the multiplicity of sports $(\mathrm{p}<$ 0.026). Hence, it can be said that a higher percentage of athletes in low-risk and mediumrisk sport disciplines were single-sport athletes, and a more significant percentage of athletes in high-risk disciplines participated in multiple sports.

Table 3 shows the mean hours of practice among low, medium, and high-risk sport disciplines in the 6 to 12 year age band.

Results of the one-way ANOVA (see Table 3) revealed that the hours of deliberate practice $(\mathrm{p}<$
$0.001)$ and physical preparation activities $(\mathrm{p}=$ 0.002 ) in the age range of 6-12 years were significantly different between the three groups.

Post hoc analyses using the Scheffe post hoc criterion for significance indicated that the mean hours of deliberate practice of participants in lowrisk sport disciplines was significantly lower than that in both medium-risk $(\mathrm{p}=0.020)$ and high-risk $(p<0.001)$ sports disciplines, but there was no significant difference between medium-risk and high-risk sport disciplines $(\mathrm{p}=0.196)$. Also, the mean physical preparation activities of participants on low-risk sport disciplines were significantly lower than those in high-risk sport disciplines $(\mathrm{p}=$ 0.002). However, there was no significant difference between medium-risk and high-risk sport disciplines $(\mathrm{p}=0.161)$, and between low-risk and medium-risk sport disciplines $(\mathrm{p}=0.211)$.

Table 2. Distribution of the Study Sample, Based on the Multiplicity of Sports

\begin{tabular}{lcccccc}
\hline Sport Discipline & \multicolumn{2}{c}{ Single-Sport } & \multicolumn{2}{c}{ Multi-Sport } & \multicolumn{2}{c}{ Total } \\
\cline { 2 - 7 } & F & P & F & P & F & P \\
\hline Low-risk & 37 & 62.7 & 22 & 37.3 & 59 & 100.0 \\
Medium-risk & 37 & 52.9 & 33 & 47.1 & 70 & 100.0 \\
High-risk & 22 & 37.9 & 36 & 62.1 & 58 & 100.0 \\
\hline
\end{tabular}

Table 3. ANOVA to Compare the Mean Hours of Practice in the 6 to 12 Year Age Band

\begin{tabular}{|c|c|c|c|c|c|}
\hline Variable & $\mathbf{N}$ & Mean & Std. Deviation & $\mathbf{F}$ & p \\
\hline Hours of deliberate play & & & & 2.092 & 0.126 \\
\hline Low-risk & 59 & 118.98 & 28.47 & & \\
\hline Medium-risk & 70 & 100.84 & 31.06 & & \\
\hline High-risk & 58 & 41.24 & 18.61 & & \\
\hline Hours of deliberate practice & & & & 10.083 & $>0.001$ \\
\hline Low-risk & 59 & 1058.29 & 211.62 & & \\
\hline Medium-risk & 70 & 508.67 & 111.79 & & \\
\hline High-risk & 58 & 155.55 & 49.28 & & \\
\hline Hours of physical preparation & & & & 6.253 & 0.002 \\
\hline Low-risk & 59 & 263.08 & 59.24 & & \\
\hline Medium-risk & 70 & 149.80 & 49.11 & & \\
\hline High-risk & 58 & 26.45 & 11.66 & & \\
\hline Hours of mental preparation & & & & 2.439 & 0.090 \\
\hline Low-risk & 59 & 52.00 & 25.95 & & \\
\hline Medium-risk & 70 & 23.77 & 11.03 & & \\
\hline High-risk & 58 & 0.90 & 0.90 & & \\
\hline
\end{tabular}

Table 4 shows the mean hours of practice among low, medium, and high-risk sport disciplines in the 13 to 15 year age band. The oneway ANOVA revealed no significant difference in the mean hours of practice among low, medium, and high-risk sport disciplines ( $\mathrm{p}>0.05$ ). Table 5 shows the mean hours of practice among low, medium, and high-risk sport disciplines in the age band above 16 .

Results of one way ANOVA in Table 5 revealed that the hours of deliberate play $(\mathrm{p}=$ $0.015)$, deliberate practice $(\mathrm{p}=0.010)$, physical preparation $(\mathrm{p}=0.003)$ and mental preparation $(\mathrm{p}$ $=0.019$ ), activities in the age band above 16 years 
were significant difference between the three groups.

Post hoc analyses using the Scheffe post hoc criterion for significance indicate that the mean hours of deliberate play of participants in lowrisk sport disciplines were significantly lower than that in high-risk sport disciplines $(\mathrm{p}=$ 0.028). However, there was no significant difference between medium-risk and high-risk sport disciplines $(p=0.907)$, and between lowrisk and medium-risk sport disciplines $(\mathrm{p}=$ 0.060 ).

Table 4. ANOVA to Compare the Mean Hours of Practice in the 13 to 15 Year age Band

\begin{tabular}{|c|c|c|c|c|c|}
\hline Variable & $\mathbf{N}$ & Mean & Std. Deviation & $\mathbf{F}$ & $\mathbf{p}$ \\
\hline Hours of deliberate play & & & & 2.967 & 0.054 \\
\hline Low-risk & 59 & 36.07 & 12.14 & & \\
\hline Medium-risk & 70 & 174.57 & 50.91 & & \\
\hline High-risk & 58 & 152.14 & 49.49 & & \\
\hline Hours of deliberate practice & & & & 0.894 & 0.411 \\
\hline Low-risk & 59 & 638.54 & 134.26 & & \\
\hline Medium-risk & 70 & 689.37 & 87.48 & & \\
\hline High-risk & 58 & 844.10 & 110.51 & & \\
\hline Hours of physical preparation & & & & 1.025 & 0.361 \\
\hline Low-risk & 59 & 201.39 & 41.11 & & \\
\hline Medium-risk & 70 & 260.45 & 42.91 & & \\
\hline High-risk & 58 & 179.76 & 41.47 & & \\
\hline Hours of mental preparation & & & & 0.060 & 0.942 \\
\hline Low-risk & 59 & 92.10 & 42.12 & & \\
\hline Medium-risk & 70 & 108.83 & 46.90 & & \\
\hline High-risk & 58 & 92.26 & 25.06 & & \\
\hline
\end{tabular}

Table 5. ANOVA to Compare the Mean Hours of Practice in the Age Band Above 16 Years

\begin{tabular}{|c|c|c|c|c|c|}
\hline Variable & $\mathbf{N}$ & Mean & Std. Deviation & $\mathbf{F}$ & Sig. \\
\hline Hours of deliberate play & & & & 4.323 & 0.015 \\
\hline Low-risk & 59 & 42.31 & 15.73 & & \\
\hline Medium-risk & 70 & 179.77 & 41.63 & & \\
\hline High-risk & 58 & 205.31 & 55.86 & & \\
\hline Hours of deliberate practice & & & & 4.762 & 0.010 \\
\hline Low-risk & 59 & 721.83 & 235.76 & & \\
\hline Medium-risk & 70 & 2008.31 & 356.47 & & \\
\hline High-risk & 58 & 1688.66 & 288.68 & & \\
\hline Hours of physical preparation & & & & 5.999 & 0.003 \\
\hline Low-risk & 59 & 248.54 & 83.28 & & \\
\hline Medium-risk & 70 & 816.25 & 129.36 & & \\
\hline High-risk & 58 & 542.41 & 127.56 & & \\
\hline Hours of mental preparation & & & & 4.068 & 0.019 \\
\hline Low-risk & 59 & 41.42 & 21.03 & & \\
\hline Medium-risk & 70 & 242.73 & 69.28 & & \\
\hline High-risk & 58 & 143.45 & 37.69 & & \\
\hline
\end{tabular}

The mean hours of deliberate practice of participants in low-risk sport disciplines were significantly lower than that in both medium-risk $(\mathrm{p}=0.012)$ and high-risk $(\mathrm{p}=0.015)$ sports disciplines, but there was no significant difference between medium-risk and high-risk sport disciplines $(\mathrm{p}=0.759)$.

The mean hours of physical preparation of participants in low-risk sport disciplines were significantly lower than those in medium-risk sport disciplines $(p=0.003)$. However, there was no significant difference between medium-risk and high-risk sport disciplines $(\mathrm{p}=0.254)$ and between low and high-risk sport disciplines $(\mathrm{p}=0.233)$.

The mean hours of mental preparation of participants in low-risk sport disciplines were significantly lower than that in medium-risk sport disciplines $(p=0.019)$. However, there was no significant difference between medium-risk and high-risk sport disciplines $(\mathrm{p}=0.378)$ and between low and high-risk sport disciplines $(\mathrm{p}=0.378)$.

\section{DISCUSSION}

It seems that the amount and type of practice vary depending on the type of sport being 
practiced (13). Accordingly, the present study aimed to investigate the history of training activities among expert athletes of different sport disciplines with varying degrees of risk.

The findings of the present study showed that the age of beginning the sport varied depending on the level of risk associated with the sport. The age of initial participation in the sport was significantly lower in low-risk sport disciplines than medium and high-risk sport disciplines. The age of beginning the sport was lower in mediumrisk sport disciplines than in high-risk sport disciplines. It seems that the age of beginning the sport in its specialized form depends on several factors such as the physical, physiological, cognitive, psychological needs of that sport in addition to the motor abilities possessed by the athlete.

Motor abilities are contextual and fundamental components of motor skills. Through carrying out many perceptual-motor tests, Fleishman (24) identified several distinct human motor abilities. According to him, there are eleven perceptualmotor abilities, including multi limb coordination, control precision, response orientation, reaction time, speed of arm movement, rate control, manual dexterity, finger dexterity, arm-hand steadiness, wrist/finger speed, and aiming. Furthermore, Fleishman identified nine physical proficiency abilities, including static strength, dynamic strength, explosive strength, trunk strength, flexibility, dynamic flexibility, gross body coordination, and fit stamina (to which most people refer as physical preparation). According to various studies, perceptual-motor abilities and physical proficiency required across sports are different (25).

Additionally, sports skills differ in terms of their underlying motor abilities; they also differ in terms of the cognitive demands of the sport participant. Some sport skills consume few cognitive resources while other sport skills depend on the speed of one's mental ability to solve a problem and process relevant information to successfully perform the task. Also, the biochemical demand, and cardiorespiratory and neuromuscular coordination of different sports vary widely, and the psychological needs of sports are also different (26). As a result, athletes that participate in stress-inducing sports must be trained to properly deal with such psychological variables (26).
Overall, it seems that differences between the ages of beginning low, medium, and high-risk sports depend on the different physical, physiological, motor, cognitive, and psychological demands required by that sport. The age of beginning the sport may also depend on the age of reaching peak athletic performance. Some studies have estimated the age of peak athletic performance to be different across sports (27). The present study estimated the age of reaching peak athletic performance to be 16.87 years in low-risk sport disciplines (e.g., gymnastics and swimming), 21.78 years in medium-risk sport disciplines (e.g., karate and wushu), and 22.32 years in high-risk disciplines (e.g., rock climbing and paragliding). According to Ericsson et al. (13), one must have at least ten years of experience in practicing a specialized discipline to reach peak athletic performance. As a result, the difference between the ages of beginning low, medium, and high-risk sport disciplines in our study explains why athletes in the respective sports reach peak performance at different ages.

Another finding revealed in the present study showed differences among low, medium, and high-risk disciplines in terms of the multiplicity of sport participation. In low and medium-risk disciplines, training hours of other sports were less, and the athletes were more likely to participate in only one sport. However, high-risk sports athletes were much more likely to participate in multiple sports. Perhaps one possible explanation for this observation is that the age of beginning the sport was greatest in high-risk sport disciplines. Thus athletes had more time to participate in additional sports and comprehensive training as a child.

In addition to the age of beginning the sport and age of reaching peak athletic performance, the amount of deliberate practice and physical preparation activities in the three types of sports were different in the three stages of sampling, specializing, and investing in terms of the degree of risk. In the age range of 6-12 years, the hours of deliberate practice and physical preparation activities were more in low-risk sport disciplines than in other sport disciplines. Whereas, at the investing stage (over 16 years of age), the hours of deliberate practice and physical preparation activities were more in medium and high-risk sport disciplines than in low-risk sport disciplines. Also, the difference in the amount of 
practice can be attributed to the differences between the physical, physiological, cognitive, and psychological needs of the different sports. For instance, Soberlak and Côté (16) showed in a study that professional ice hockey players spent more than 10,000 hours from the ages of 6 to 20 years participating in sports. Within the allotment of 10,000 hours, about 2,300 hours were spent participating in sports other than hockey, 350 hours were dedicated to playing deliberate games other than hockey, more than 3,000 hours were dedicated to deliberate hockey practice 2,400 hours were involved in deliberate hockey games. The significant finding in the study conducted by Soberlak and Côté (16) was that a large amount of deliberate play and participation in other sports had occurred before the age of 15. A large amount of deliberate practice had occurred after the age of 15 . This contrasts with a study conducted by Tan and Low (28) investigating practice allocations within swimmers. They concluded that a large part of the elite swimmers' activities was allocated to deliberate practice (i.e., more than 5000 hours), while other activities such as deliberate play were less than 1000 hours. This shows that elite swimmers have not allocated much time to voluntary and inherently motivated swimming activities.

Another finding of the present study showed no statistically significant difference between the athletes of the low, medium, and high-risk sport disciplines in terms of the average hours of training mental skills in the 6 to 12 years and 13 to 15 years. One of the possible reasons was the small amount of training mental skills services offered to the athletes. Our findings revealed that the amount of mental skills training in the six sports disciplines was 25 . Three hours on average in the 6 to 12-year age range, 97.73 hours in the 13 to 15 -year age range, and 142.53 hours in the over 16-year age range. By reviewing 45 different studies, Weinberg and Williams (29) concluded that 38 cases (i.e., $85 \%$ of the cases being reviewed) were indicative of the positive effect of training mental skills on performance. Despite the benefits of mental training skills, Weinberg and Williams (29) reported that many athletes and coaches do not actively engage in mental skills training practices. The authors indicated that this is unawareness of how to teach and train mental skills, the belief that psychological characteristics are inherent and unchangeable, and the lack of time to devote to mental skills training. Hence, it seems that we must build a culture regarding this case to highlight the importance of dealing with mental skills for athletes, coaches, and authorities.

\section{CONCLUSION}

In conclusion, the findings of this study show that the age of beginning a sport, the multiplicity of sport participation, as well as the amount of deliberate practice and deliberate play within the three age ranges of 6 to 12,13 to 15 , and above 15 years of age, depends on the type of sports being practiced.

We observed that athletes involved in all of these sports trained mental skills to a much lesser extent than training for the sport. Given the importance of mental skills in the progress of athletic performance, it is suggested that more attention is paid to training and practicing mental skills in addition to practicing the sports discipline.

\section{APPLICABLE REMARKS}

- Coaches explain to athletes the importance of psychological skills in achieving high levels of performance.

- Coaches should note that the age of beginning a sport depends on the type of sports being practiced.

\section{REFERENCES}

1. Davids K, Baker J. Genes, environment and sport performance: why the nature-nurture dualism is no longer relevant. Sports Med. 2007;37(11):961-980. doi: 10.2165/00007256-200737110-00004 pmid: 17953467

2. Gray B, Semsarian C. Utility of genetic testing in athletes. Clin Cardiol. 2020;43(8):915-920. doi: 10.1002/clc. 23289 pmid: 31925963

3. Mugandani SC. Athletic Performance enhancing ACE, ACTN3, AMPD1 Genetic markers, Fitness characteristics, C-Reactive Protein and Uric Acid of Cricket, Netball, Rugby and Soccer Players: A review. J Appl Sport Sci. 2019;1:131-149. doi: 10.37393/jass.2019.01.13

4. Ericsson KA, Harwell KW. Deliberate Practice and Proposed Limits on the Effects of Practice on the Acquisition of Expert Performance: Why the Original Definition Matters and Recommendations for Future Research. Front Psychol. 2019;10:2396. doi: 10.3389/fpsyg.2019.02396 pmid: 31708836 
5. Ericsson KA. Towards a science of the acquisition of expert performance in sports: Clarifying the differences between deliberate practice and other types of practice. J Sports Sci. 2020;38(2):159-176. doi: 10.1080/02640414.2019.1688618 pmid: 31718526

6. Thomas CE, Gastin PB, Abbott G, Main LC. Impact of the talent development environment on the wellneing and burnout of caribbean youth track and field athletes. Europe $J$ Sport Sci. 2020:1-29. doi: $10.1080 / 17461391.2020 .1775894$

7. Wilkinson SG, Grecic D. Talent development for professional rugby league: observations and analysis from a career in rugby's high-performance environment. J Qualit Res Sport Stud. 2019;13(1):135-174.

8. Gruber H, Jansen P, Marienhagen J, Altenmueller E. Excellence. Adaptions during the acquisition of expertise. Talent Develop Excellence. 2010(1):3-15.

9. Côté J, Fraser-Thomas J. Play, practice, and athlete development. Developing sport expertise: Routledge2007.

10. Côté J, Baker J. Practice and play in the development of sport expertise. Hosp. 2007;3:184-202. doi: 10.1002/9781118270011.ch8

11. Côté JJ. The influence of the family in the development of talent in sport. Sport Psychol. 1999;13(4):395-417. doi: 10.1123/tsp.13.4.395

12. Côté J, Hay J. Children's involvement in Sport: A developmental perspective. In. JM Silva \& DE Stevens (Eds.), Psychological foundations of sport. Boston, MA: Allyn \& Bacon; 2002.

13. Ericsson KA, Krampe RT, Tesch-Römer CJPr. The role of deliberate practice in the acquisition of expert performance. Personal Individ Differ. 1993;100(3):363. doi: 10.1037/0033-295X.100.3.363

14. Kirk DJ. Physical education, youth sport and lifelong participation: the importance of early learning experiences. Europe Physic Educat Rev. 2005;11(3):239-255. doi: 10.1177/1356336X05056649

15. Baker J, Cote J, Abernethy B. Learning from the experts: practice activities of expert decision makers in sport. Res Q Exerc Sport. 2003;74(3):342-347. doi: 10.1080/02701367.2003.10609101 pmid: 14510301

16. Soberlak P, Cote JJ. The developmental activities of elite ice hockey players. J Appl Sport Psychol. 2003;15(1):4149. doi: 10.1080/10413200305401

17. Baker J, Cote J, Abernethy BJ. Sport-specific practice and the development of expert decision-making in team ball sports. J Appl Sport Psychol. 2003;15(1):12-25. doi: 10.1080/10413200305400

18. Buckley PS, Bishop M, Kane P, Ciccotti MC, Selverian S, Exume D, et al. Early Single-Sport Specialization: A Survey of 3090 High School, Collegiate, and Professional Athletes. Orthop J Sports Med. 2017;5(7):2325967117703944. doi: 10.1177/2325967117703944 pmid: 28812031

19.Zuckerman MJP. Sensation seeking and sports. Personal Individ Differ. 1983;3(3):285-292. doi: 10.1016/01918869(83)90150-2

20. Hopwood M. The Developmental History of Athletes Questionnaire: Towards a comprehensive understanding of the development of sport expertise: Victoria University; 2013.

21. Berry J, Abernethy B, Cote J. The contribution of structured activity and deliberate play to the development of expert perceptual and decision-making skill. $J$ Sport Exerc Psychol. 2008;30(6):685-708. doi: 10.1123/jsep.30.6.685 pmid: 19164836

22. Memmert D, Baker J, Bertsch CJ. Play and practice in the development of sport-specific creativity in team ball sports. High Abilit Stud. 2010;21(1):3-18. doi: 10.1080/13598139.2010.488083

23. Oldenziel K, Gulbin J, Gagne FJASC. How do elite athletes develop? A look through the rear-view mirror. Canberra. 2003.

24. Theologus GC, Fleishman EA. Development of a taxonomy of human performance: Validation study of ability scales for classifying human tasks. America Institut Res Pittsburgh PA. 1971. doi: 10.21236/AD0736194

25. Magill RA, Anderson DI. Motor learning and control: Concepts and applications. McGraw-Hill New York2007.

26. Bompa TO, Buzzichelli C. Periodization: theory and methodology of training: Human kinetics; 2018.

27. Allen SV, Hopkins WG. Age of Peak Competitive Performance of Elite Athletes: A Systematic Review. Sports Med. 2015;45(10):1431-1441. doi: 10.1007/s40279-015-0354-3 pmid: 26088954

28. Tan LSA, Low FLJJ. Developmental practice activities of elite youth swimmers. Malaysia J Move Health Exercise. 2014;3. doi: 10.15282/mohe.v3i0.15

29. Weinberg R, Williams JJ. Integrating and implementing a psychological skills training program. AspPgtpp. 2001;4:347-377. 